\title{
Texture Feature Extraction through Oblong Aperture and Segmentation using Level Sets
}

\author{
K M Sadyojatha \\ Ballari Institute of Technology \\ and Management, Bellary, \\ Karnataka, India
}

\author{
Vinayadatt $\mathrm{V}$ Kohir \\ PDA college of Engineering, \\ Gulbarga, \\ Karnataka, India,
}

\author{
Subhash Kulkarni \\ Jayaprakash Narayan College \\ of Engineering, Mahabubnagar, \\ AP, India
}

\begin{abstract}
An explorative work on texture feature extraction through oblong aperture for the non random type of texture images is presented in this paper. These features are further useful for segmenting the texture regions using the level set framework. The statistical moment descriptors are obtained within a small aperture and are embedded into a level set frame work for segmentation. The shape of the aperture normally would be square and size selection done optimally. The square shaped aperture sometimes does not yield good feature descriptors particularly when the textured regions are highly structured. The proposed oblong aperture provides an appreciable change in extracted features particularly for structured images in contrast to the square shaped aperture.
\end{abstract}

\section{General Terms}

Random textures, image processing

\section{Keywords}

Textures, Moments, Level sets, oblong aperture

\section{INTRODUCTION}

Texture segmentation aims at discriminating between regions which have different textures. Texture representation and modeling can be roughly divided into two categories: Statistical based approach and Filtering based approach [5]. Statistical modeling is based on the assumption that each texture has unique statistical attribute. The filtering model is based on applying filter bank to the image and considering the filter's response has information about the local behavior of the image. Popular choice is the Gabor filters. This paper is poised on using moment descriptors viz. mean, standard deviation and $3^{\text {rd }}$ order moment of texture images over a predefined neighborhood [6]. Further, the level sets method is used on the derived feature image for extracting the homogeneous regions. Further this method can be less time consuming because of using of fast level sets [7].

Here a particular case of segmentation of structured textures is explored by using an oblong aperture, as the structured textures contain high correlation between pixels and directionality is obvious. The moment features extracted through such an aperture are embedded into level set frame work for segmentation. Based on an earlier work by Chan and Vese [8], the level set technique is extended to texture segmentation with a much more simplified version. The advantage of Chan and Vese model being (a) allows arbitrary initialization of the contour (b) depending upon a particular feature derived from the image it allows for boundary detection (c) It avoids re-initialization of intermediate contours during propagation. The model is similar to Mumford-Shah [4] segmentation functional processing level sets formulation.

\section{FEATURE EXTRACTION THROUGH OBLONG APERTURE}

Achieving better texture image segmentation results depends partly on the discriminating capability of the segmentation algorithm and partly on the information provided by the feature descriptors regarding the similarities and dissimilarities among the regions of same and different textures respectively. The size of the aperture should be selected in such a way that the information of the spatial gray level dependencies obtained should allow to distinguish between the different textural regions. Therefore the size of the aperture should be selected circumspectly. Normally a square shaped aperture with an appropriate size is used around each pixel and overlapped on every pixel to obtain the required features. Feature thus obtained must be a computed data which should yield a single value capable of representing the texture content within the aperture. The square aperture by and large gives better features except for some of the non random (structured) textures like brick wall, floor tiled with paving stones type of the images. The structured textures are the ones which exhibit strong correlation between pixels. Possibility of extracting features for these previously said images through an oblong aperture is explored and the promising results are presented in this paper. Some of such images from Brodatz album are mixed to form two layered textures as shown in fig 1 .
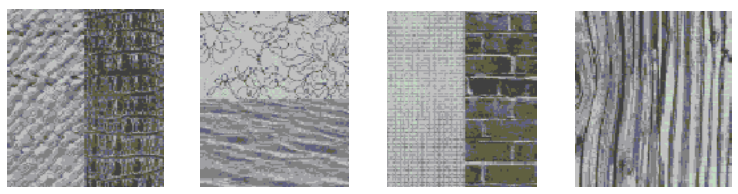

Fig 1: Sample combinations of structured textures from Brodatz album

The statistical moments are used here as the feature energy terms extracted around every pixel within a local area called aperture of a suitable size. Generally a square shaped aperture is used [6] but here an oblong aperture is used as a special case for extracting the features of structured textures. Some of the results obtained with square aperture are presented below 
where the segmentation is not very proper. For such images the shape of the window is stretched, invariably either horizontally or vertically in the direction of orientation of texture population. As the textures used for experimentation are highly structured, having strong correlation between pixels and as directionality is obvious in such images; it becomes necessary to stretch the aperture along the direction of orientation of the texture population in order to enhance the discrimination capabilities. The aperture selected here is with a size $m_{1} \times m_{2} \cdot m_{1}>m_{2}$ when the orientation of texture population is in horizontal direction and vice versa when the orientation is in vertical direction.

\section{MOMENT DESCRIPTORS}

Selection of appropriate feature becomes an important phase of pre-segmentation. Global extraction of these features makes less sense as this will not yield any measure of the distribution of intensities with respect to their spatial local neighborhood. Therefore the features are to be extracted within a local aperture of a suitable size around every pixel. The size of the aperture needs to be selected circumspectly because the larger aperture causes uncertainties along the region boundaries and the smaller aperture perhaps may fail to capture certain variations in some of the textures. The precise descriptor and the correct size of the aperture are the imperative attributes for good texture segmentation. From the results obtained here it can also be concluded that, like the size of aperture and correct descriptor, the shape of the aperture is equally important in proper segmentation of the textured regions.

Generally the first moment quantifies the mean of the values of members belonging to a class. The second moment measures the deviation of the values of class members from their mean. It indicates the spread of the distribution around the mean. The third moment or the skew measures the nonsymmetry of distribution of members around the mean. The computation of the moments is done within the aperture selected whose dimensions are $\mathrm{m}_{1}$ and $\mathrm{m}_{2}$ as below.

The first moment is calculated as,

$\bar{x}=\frac{1}{m_{1} * m_{2}} \sum_{i=0}^{m_{1}-1} \sum_{j=0}^{m_{2}-1}(x(i, j))$

And the second moment as,

$y=\left[\frac{1}{\left(m_{1} * m_{2}\right)-1} \sum_{i=0}^{m_{1}-1} \sum_{j=0}^{m_{2}-1}(x(i, j)-\bar{x})^{2}\right]^{\frac{1}{2}}$

Where $m_{1}>m_{2}$ when the orientation of the texture population is horizontally distributed and $\mathrm{m}_{1}<\mathrm{m}_{2}$ when the orientation of the texture population is vertically distributed. In general the $\mathrm{n}^{\text {th }}$ moment is computed as.
$m_{n}=E[x-\mu]^{k}$ Within the aperture of $\mathrm{m}_{1} * \mathrm{~m}_{2}$

The features thus selected present a favorable ambience for segmenting an image. The conventional segmentation tool works well when the between class scatter is higher than a threshold level forcing the segmentation result to be erroneous. One solution to the problem is to include spatial neighborhood information along with the feature selected.

The inevitable option is to use deformable model as a tool

\section{LEVEL SETS}

The basic idea of geometric deformable model is to couple the speed of the deforming curve with the image data, so that the evolution of the curve halts at the object boundaries. The level set is defined as the set of points that has the same function values. Instead of tracking a curve through time, as in parametric deformable model, the level set method evolves a curve by updating the level set function at fixed co-ordinates through time.

In level set approach the evolving curve is represented by zeros and is called zero level set (ZLS). The other points in the domain represent their distances from the ZLS, i.e. $\Phi(x, y)$ $= \pm \mathrm{d}$, where $\mathrm{d}$ is the distance of the point $(\mathrm{x}, \mathrm{y})$ in the domain from the ZLS. The sign represents whether the point is inside or outside of the ZLS. The initial level set function is defined through distance mapping

In this proposed work the level set frame work proposed by Chan-Vese, which models Mumford-Shah's piecewise constant approach is used, in which the curve evolution is bidirectional allowing one the freedom of arbitrarily defining the initial curve[4],[7]. This model is region based and under steady state, segments the image into two homogeneous regions-insides and outside of the deforming curve and hence doesn't compel the level set definition through Euclidian distances. This work uses city-block distances through DSFT for defining the level set function, whose time complexity is extremely small and at the same time it is curve invariant, i.e., Independent of the size of the initial curve. This inherent property encourages the random initialization of the model curve.

The higher dimensional energy functional [6] used here is

$$
\begin{aligned}
& E\left(c_{i}, c_{o}, C\right)=\lambda_{i} \int_{\Omega}\left|I-c_{i}\right|^{2} H(\phi) d \Omega+\lambda_{i} \int_{\Omega}\left|I-c_{o}\right|^{2} \\
& (1-H(\phi)) d \Omega+\mu \int_{\Omega} \delta(\phi)|\nabla \phi| d \Omega+v \int_{\Omega} H(\phi) d \Omega
\end{aligned}
$$

This functional in equation (1) when subjected to minimization via Eulerian converts into motion PDE resulting into propagation of model curve. 
The functional in equation (4) is solved for infimum using the principles of calculus of variation. Thus the Eulerian of the energy functional in implicit level set form is reduced to

$$
\frac{\partial \phi}{d t}=\left[\begin{array}{l}
\mu \nabla \cdot\left(\frac{\nabla \phi}{|\nabla \phi|}\right)+\alpha_{1}\left(I_{r e f}-c_{i}\right)^{2} H(\phi) \\
+\alpha_{2}\left(I_{r e f}-c_{o}\right)^{2}(1-H(\phi))
\end{array}\right]
$$

In the above motion PDE the level set function $\Phi$ is a signed distance function representing a continuously differentiable surface. In equation (5) I is replaced by $I_{\text {ref }}$ which represents the feature described image data for the texture image as computed in section (3).

\section{RESULTS}

Some of the results obtained using the oblong aperture is presented in fig.2. The result for the same images obtained using square aperture is also shown in the last column of fig. 2 . The size of the aperture is selected in such a way that, the substantial amount of pixels concerned to each region are covered so that the moments derived from such an area will help in making that region homogeneous. Further the aperture is stretched in the direction of the orientation of the population. For example the aperture selected for the results in row 1 are with $m_{1}=3$ and $m_{2}=9$ i.e. the aperture stretched in the vertical direction. Similarly $\mathrm{m}_{1}$ and $\mathrm{m}_{2}$ for image in row 4 are 21 and 3 respectively, where the aperture is stretched horizontally. The segmentation results in the last column are obtained through the features extracted using a square aperture where it can be evidenced that the segmentation is improper.

Some of the natural textures which are highly structured are also used for segmentation with the proposed method and is successfully achieved as shown in fig.3. As an alternative to the computationally intensive methods used in [1] [2] [3], very simple moment features based frame work is used to obtain almost similar or some times better results.

\section{CONCLUSION}

Our motivation here has been to develop unified approach with simple moment features and the robust, powerful level set framework for texture segmentation. One of the particular cases of structured texture segmentation is addressed in this paper. Instead of resorting to computationally demanding Gabor features, simple moment features are used here and for extracting these features for structured textures we have adopted an oblong aperture instead of a regular square one, which has yielded promising results. The selection of the size of the aperture is still an open ended problem which is done interactively in this case.
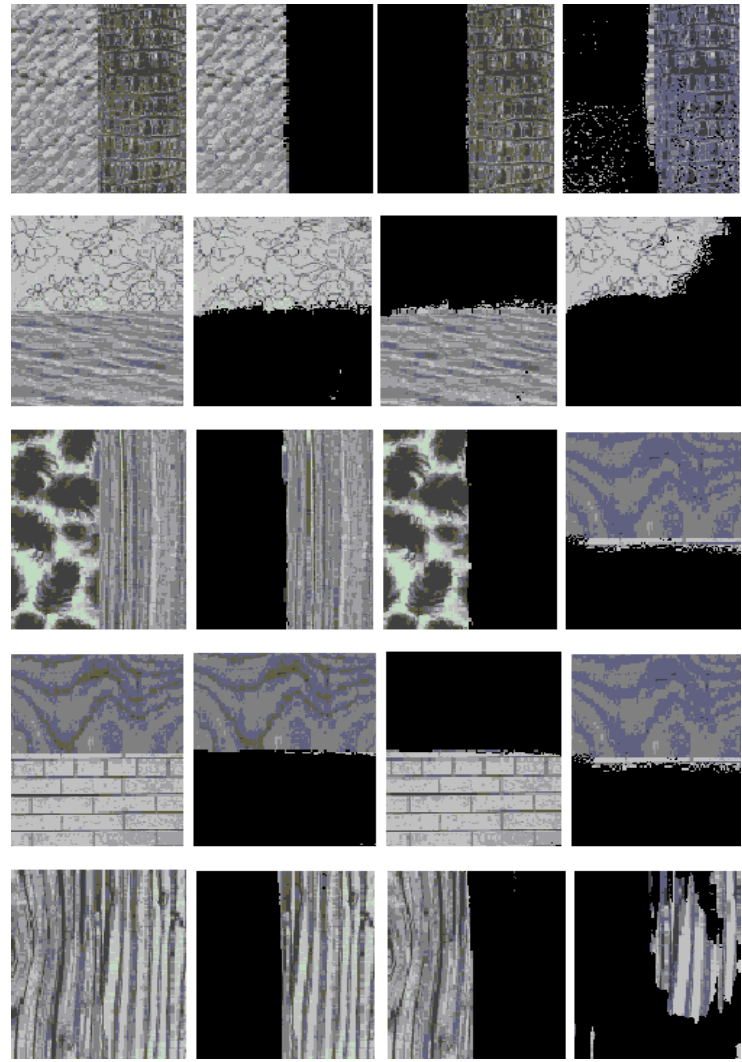

Fig 2: Column 1 indicates original images, Column $2 \& 3$ indicate segmented images with oblong aperture, Column 4 indicates segmented images with square aperture
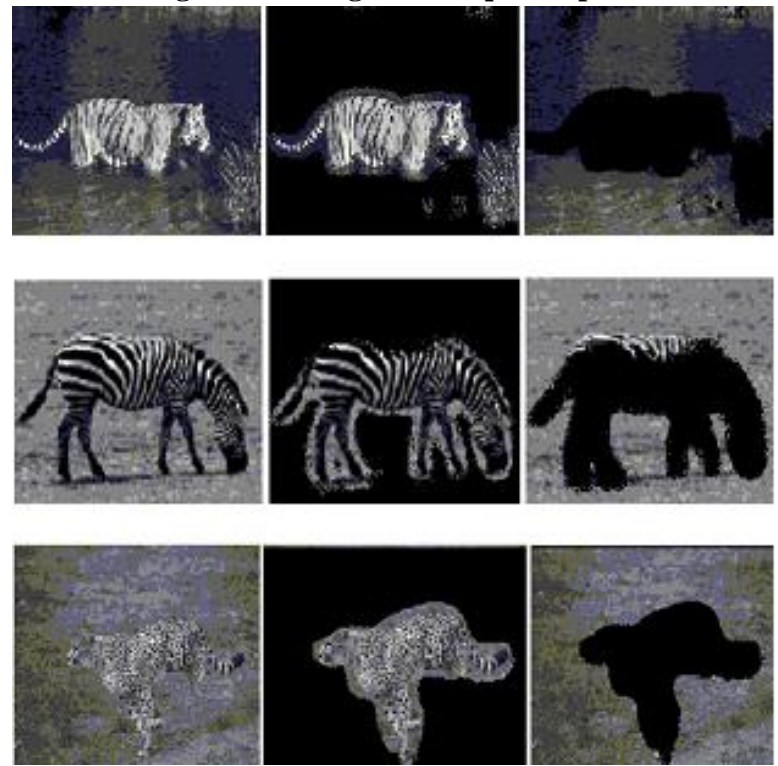

Fig 3: Segmentation results of some natural images which are highly structured. Column 1 indicates original images, Column 2\&3 indicate segmented images with oblong aperture. 


\section{REFERENCES}

[1] C. Sagiv, N. A. Sochen, and Y. Y. Zeevi. Integrated active contours for texture segmentation.IEEE Trans. Image Processing, 1:1-19, 2004

[2] GUI-SONG XIA ET AL:: Texture Segmentation by Grouping EllipseEnsembles via Active Contours, British Machine Vision Conference (BMVC) 2011

[3] N. Houhou, J. P. Thiran, and X. Bresson. Fast texture segmentation model based on the shape operator and active contour. In Proc. of Computer Vision and Pattern Recognition,2008

[4] Mumford D. and Shah J., "Optimal approximation by piece wise smooth function and associated variational problems". Commu ${ }^{\mathrm{n}}$.Pure Appl. Math,42, 1989, 577-685.
[5] R.Conners and C.Harlow, "A theoretical comparison of texture algorithms," IEEE trans. Pattern Anal. Mach. Intell, Vol.2, No.PAMI-3, pp.204-222, May 1980.

[6] Sadyojatha K.M. and Subhash Kulkarni "Texture Segmentation Using Level Sets,”, ICCR 2008, Mysore, India, 168-174, 2008.

[7] Sandeep V.M. and Subhash Kulkarni "Curve Invariant Fast Distance Mapping Technique for Level Sets," IEEE's ICSIP2006, Hubli, India, 777-780, 2006.

[8] T.F.Chan, L.A.Vese, Active contours without edges, IEEE trans. on image processing, 10, 2001, 266-276. 